\title{
A New Dynamic Model of a Two-Wheeled Two-Flexible-Beam Inverted Pendulum Robot
}

\author{
Amin Mehrvarz ${ }^{1 *}$, Mohammad Javad Khodaei ${ }^{1 *}$, William Clark ${ }^{1}$, and Nader Jalili ${ }^{1,2}$ \\ ${ }^{1}$ Department of Mechanical and Industrial Engineering, Northeastern University, Boston, MA, U.S. \\ ${ }^{2}$ Professor and Head, Department of Mechanical Engineering, University of Alabama, Tuscaloosa, AL, U.S. \\ * Co-first author
}

\begin{abstract}
Two-wheeled inverted pendulum robots are designed for self-balancing and they have remarkable advantages. In this paper, a new configuration and consequently dynamic model of one specific robot is presented and its dynamic behavior is analyzed. In this model, two cantilever beams are on the twowheeled base and they are excited by voltages to the attached piezoelectric actuators. The mathematical model of this system is obtained using the extended Hamilton's Principle. The results show that the governing equations of motion are highly nonlinear and contain several coupled terms. These complex equations are solved numerically and the natural frequencies of the system are extracted. The system is then simulated in both lateral and horizontal plan movements. This paper proposes a new model of two-wheeled two-flexible-beam inverted pendulum Robot and investigates its complex dynamic; however, the derived equations will be validated experimentally and a suitable control strategy will be applied to the system to make it fully automated and more applicable in the future works.
\end{abstract}

Index Terms-Two-wheeled robot, flexible system, EulerBernoulli beam, inverted pendulum.

\section{INTRODUCTION}

Inverted pendulums are traditional dynamic problems. If an inverted pendulum is used in a moving cart, new type of interesting problems will appear. One of these problems is two-wheeled inverted pendulum systems. Because of their small size, great performance in quick driving, and their stability with controller, scientists and engineers are interested in them [1]. Based on this interest, each year new models are introduced and new robots are made. Self-transportation systems such as hoverboards and small two-wheeled robots are the most important patents based on the moving inverted pendulums [2].

The idea of using self-transportation systems comes from a push in the transportation industry to develop transportation systems that contribute less to pollution and cause less damage to the environment overall. One approach to this has been a shift to Personal Electric Vehicles (PEVs), which are powered by electricity rather than combustion. PEVs provide many benefits to both consumers and society, including lower costs than automobiles, shorter trip times for short distances, cleaner transportation, and mobility for the disabled [3]. One popular type of PEV that has emerged in recent years is the "Stand-on Scooter". In 2005, Ulrich analyzed existing stand-on scooter technology and estimated that the light design of these PEVs combined with their modest range and speed would be "highly feasible technically, and with substantial consumer demand could be feasible economically" [3]. One type of stand-on scooter analyzed by Ulrich was the Segway, which is a type of PEV that marketed itself based on its inverted pendulum balancing mechanism and its agility.

Inverted-pendulum transporter is a type of self-balancing system that allows for an operator to control it without the need for a throttle. Instead, the device applies a lateral movement to the system based on an angle applied by the operator, who acts as an inverted pendulum, in order to keep the inverted pendulum balanced and stable in the upright position. A popular inverted pendulum PEV is the "Hoverboard", which consists of two motorized wheels connected to two independent articulating pads. The operator controls the speed of travel by leaning forward and backward and controls the direction of travel by twisting the articulating pads with their feet. This allows for an inexpensive transportation system that is compact, as it does not require any large motor or steering apparatus, and agile, as it can be controlled easily and move quickly. Considerable work has already been done in developing a transportation device that uses this sort of self-balancing mechanism. Grasser et al. developed a scaleddown prototype of a self-balancing pendulum, but not a fullscale version that could be ridden by a person [4]. Tsai et al. [5] developed a self-balancing PEV but used handlebars for guidance rather than the articulating pads like the hoverboard.

Moving inverted pendulum systems are also used in robots for different objects. For example, Solis et al. used the robot for educational purposes [6] or Double Robotics Inc made a robot for telecommuter [7]. Two steps are needed to design a proper controller for these robots. Finding a perfect model for the two-wheeled inverted pendulum is the first step and designing the best controller is the second step. It should be mentioned that the self-balancing systems like these robots create difficult control problems, as they are inherently unstable and subject to unpredictable external forces from their environment. To solve these problems, a robust dynamic model must be developed, in order to fully understand the physical properties of the system, and also a better comprehending of how to control it to maintain stable. These robots also need one or two motors for moving which their motors models should be considered in the robots dynamic [8].

Decreasing the number of wheels which are unnecessary in most of the time and only used for the system's balancing was one of the first ideas of the two-wheeled robots 
[9]. For this purpose, Kim et al. developed a mathematical model for a self-balancing two-wheeled robot that was capable of changing direction. This robot acted as a rigid single-pendulum, allowing the model to assume a lumpedparameter system and they designed a linear controller for their robot [9]. Other researchers tried to develop [1] or modify the previous models [10]. They also tried to improve the controller performance during the robots' operation. In a research, Zafar et al. discussed a derived mathematical model for a self-balancing inverted-pendulum robot and implement the operational space controller [11]. In other researches, new controllers for the two-wheeled inverted pendulum systems were designed. These controllers can work with time-varying parametric uncertainties [12], strong nonlinear behaviors due to abrupt external disturbances [13], and initial errors, pulse disturbance and random noises [14].

A new type of two-wheeled inverted pendulum robot is also designed for specific applications. This pendulum was not rigid and it behaves as a flexible system. Partial Differential Equations (PDEs) are the most standard way of mathematically representing continuous systems and has been used for vibrational analysis and control for different models and applications $[15]-[18]$. Researchers work on the flexible structure such as beams and bars with the base motion for several years but they did not consider their systems as a moving robots. They used flexible structures with moving base for different applications, such as micro gyroscopes and piezoresponse force microscopy and etc. [19]-[21]. The base motion creates different accelerations and complex nonlinear PDE equations of motion for the flexible system. The first idea of using flexible structure as an inverted pendulum in robot was created by Nguyen et al. They studied a linearized mathematical model and controller for a single flexible inverted pendulum, this model only accounted for lateral movement in one direction rather than in the two directions and it has four wheels [22]. In another robot Mehrvarz et al. modeled a two-wheeled flexible inverted pendulum which can move in one direction [23]. They also designed a MPC controller for their robot and showed that the percision of their modeling [24]. Their robot cannot move in the plane and this was their main problem.

Here, to solve the problem of two-wheeled flexible beam inverted pendulum which is used in [23], two-wheeled twoflexible beam inverted pendulum model is addressed. This robot is designed to move in-plane and has not the previous problem. Because of its complexity and non-linearity, the dynamic model and the vibrations of the system are just considered and analyzed in this paper and controller is not designed for this system right now. The proposed model analyzes the pure dynamics of the robot and the vibrations of the beams at the same time, which is a novel approach. The main goal of this paper is to investigate and simulate the dynamic model of a piezoelectrically actuated cantilever beams on the two-wheeled robot.

The remainder of this paper is organized as follow. The dynamic equations of the system are derived in Section II and a brief summary of their solution is presented in Section

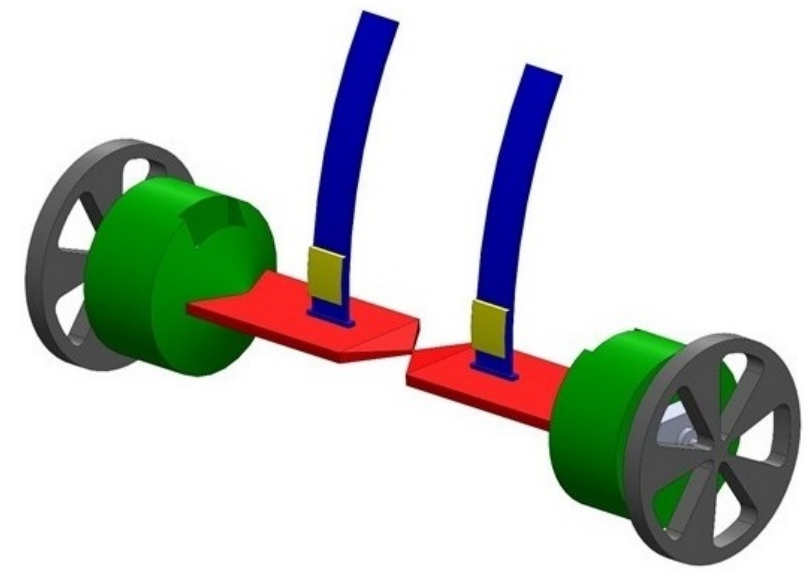

Fig. 1. The proposed dynamic model of the two-wheeled two-flexible-beam inverted pendulum robot.

IIII In Section IV the simulation results are discussed and a conclusion is given in Section $\mathrm{V}$.

\section{MathematicAL MODELING}

In this section, the governing equations of motion are derived using the extended Hamilton's principle. In order to apply the extended Hamilton's principle to the system, the positions and translational and rotational velocities of these elements are needed to be defined mathematically. As shown in Fig 1, the system has two beams and two piezoelectric actuators to excite the beams. These beams are mounted on two independent bases, which are attached to two wheels and DC motors. In this model, as seen in Fig 1, two flexible cantilever beams act as flexible inverted pendulums fixed to two articulating bases. The mathematical model for the system represents the response of the system to small disturbances from piezoelectric actuators mounted to the base of each pendulum. These actuators cause deformation and bending in the continuous pendulums when voltage is applied to them. As seen in Fig 2, the position of the right $\left(\right.$ beam $\left._{1}\right)$ and left $\left(\right.$ beam $\left._{2}\right)$ beams in the $X Y Z$ frame are as:

$$
\left[\begin{array}{c}
X_{\text {beam }_{1}} \\
Y_{\text {beam }} \\
Z_{\text {beam }_{1}}
\end{array}\right]=\left[\begin{array}{c}
X_{c}+a \sin \varphi+\cos \varphi\left(\cos \theta_{1} w_{1}+x_{1} \sin \theta_{1}\right) \\
Y_{c}-a \cos \varphi+\sin \varphi\left(\cos \theta_{1} w_{1}+x_{1} \sin \theta_{1}\right) \\
r_{w}-\sin \theta_{1} w_{1}+x_{1} \cos \theta_{1}
\end{array}\right]
$$

$$
\left[\begin{array}{c}
X_{\text {beam }} \\
Y_{\text {beam }} \\
Z_{\text {beam }_{2}}
\end{array}\right]=\left[\begin{array}{c}
X_{c}-a \sin \varphi+\cos \varphi\left(\cos \theta_{2} w_{2}+x_{2} \sin \theta_{2}\right) \\
Y_{c}+a \cos \varphi+\sin \varphi\left(\cos \theta_{2} w_{2}+x_{2} \sin \theta_{2}\right) \\
r_{w}-\sin \theta_{2} w_{2}+x_{2} \cos \theta_{2}
\end{array}\right]
$$

where $X_{c}$ and $Y_{c}$ are the positions of the center of gravity of the robot and $a$ denotes the distance between the center of the beams and the center of gravity in the $y$-direction. The robot can rotate around the $z$-axis and the bases have different rotations around the $y$-axis. These rotational angles are shown by $\varphi, \theta_{1}$ and $\theta_{2}$. In (1) and (2), the parameters $w_{1}$ 
and $w_{2}$ represent the bending deflections of the beams and $r_{w}$ is the radius of the wheels. Since the beams are supposed to be continuous, the position of each particle in the beams are given by $x_{1}$ and $x_{2}$. The position of the right (wheel ${ }_{1}$ and base $_{1}$ ) and left (wheel $l_{2}$ and base ${ }_{2}$ ) wheels and bases can be obtained as:

$$
\begin{aligned}
& {\left[\begin{array}{c}
X_{\text {wheel }_{1}} \\
Y_{\text {wheel }_{1}} \\
Z_{\text {wheel }_{1}}
\end{array}\right]=\left[\begin{array}{c}
X_{c}+2 a \sin \varphi \\
Y_{c}-2 a \cos \varphi \\
r_{w}
\end{array}\right]} \\
& {\left[\begin{array}{c}
X_{\text {wheel }_{2}} \\
Y_{\text {wheel }_{2}} \\
Z_{\text {wheel }_{2}}
\end{array}\right]=\left[\begin{array}{c}
X_{c}-2 a \sin \varphi \\
Y_{c}+2 a \cos \varphi \\
r_{w}
\end{array}\right]} \\
& {\left[\begin{array}{c}
X_{\text {base }_{1}} \\
Y_{\text {base }_{1}} \\
Z_{\text {base }_{1}}
\end{array}\right]=\left[\begin{array}{c}
X_{c}+a \sin \varphi \\
Y_{c}-a \cos \varphi \\
r_{w}
\end{array}\right]} \\
& {\left[\begin{array}{c}
X_{\text {base }_{2}} \\
Y_{\text {base }_{2}} \\
Z_{\text {base }_{2}}
\end{array}\right]=\left[\begin{array}{c}
X_{c}-a \sin \varphi \\
Y_{c}+a \cos \varphi \\
r_{w}
\end{array}\right]}
\end{aligned}
$$

Consequently, the translational velocity of all the elements can be obtained by applying a time derivation operator to (1) through (6).

It is assumed that the system move without slippage in $y$ and $x$ directions. Hence, velocity of the center of gravity of the system can have the following relationship,

$$
\frac{\dot{Y}_{c}}{\dot{X}_{c}}=-\tan \varphi
$$

It should be noted that the whole velocity of the beams can be calculated through integration of the each beam's particles velocity along the beam. Besides the translational motion, the elements of the robot have also some rotational movements. The rotational velocity of each element can be written as:

$$
\begin{aligned}
& {\left[\begin{array}{c}
\omega_{x_{\text {wheel }_{1}}} \\
\omega_{y_{\text {wheel }_{1}}} \\
\omega_{z_{\text {wheel }_{1}}}
\end{array}\right]=\left[\begin{array}{c}
0 \\
\frac{\dot{X}_{c} \cos \varphi+\dot{Y}_{c} \sin \varphi+2 a \dot{\varphi}}{r_{w}} \\
\dot{\varphi}
\end{array}\right]} \\
& {\left[\begin{array}{c}
\omega_{x_{\text {wheel }_{2}}} \\
\omega_{y_{\text {whee }_{2}}} \\
\omega_{z_{\text {wheel }_{2}}}
\end{array}\right]=\left[\begin{array}{c}
0 \\
\frac{\dot{X}_{c} \cos \varphi+\dot{Y}_{c} \sin \varphi-2 a \dot{\varphi}}{r_{w}} \\
\dot{\varphi}
\end{array}\right]} \\
& {\left[\begin{array}{c}
\omega_{x_{\text {base }_{1}}} \\
\omega_{\text {base }_{1}} \\
\omega_{\text {base }_{1}}
\end{array}\right]=\left[\begin{array}{c}
0 \\
\dot{\theta}_{1} \\
\dot{\varphi}
\end{array}\right]} \\
& {\left[\begin{array}{c}
\omega_{x_{\text {base }_{2}}} \\
\omega_{y_{\text {base }_{2}}} \\
\omega_{z_{\text {base }_{2}}}
\end{array}\right]=\left[\begin{array}{c}
0 \\
\dot{\theta}_{2} \\
\dot{\varphi}
\end{array}\right]} \\
& {\left[\begin{array}{c}
\omega_{x_{\text {beam }}} \\
\omega_{y_{\text {beam }}} \\
\omega_{z_{\text {beam }}}
\end{array}\right]=\left[\begin{array}{c}
\dot{\varphi} \cos \theta_{1} \\
\dot{\theta}_{1}+\frac{\partial^{2} w_{1}}{\partial x \partial t} \\
\dot{\varphi} \sin \theta_{1}
\end{array}\right]}
\end{aligned}
$$

$$
\left[\begin{array}{c}
\omega_{x_{\text {beam }}} \\
\omega_{y_{\text {beam }}} \\
\omega_{z_{\text {beam }}}
\end{array}\right]=\left[\begin{array}{c}
\dot{\varphi} \cos \theta_{2} \\
\dot{\theta}_{2}+\frac{\partial^{2} w_{2}}{\partial x \partial t} \\
\dot{\varphi} \sin \theta_{2}
\end{array}\right]
$$

Since the system has 6 different parts, the kinetic energy of the whole system including the translational and rotational parts can be obtained as:

$$
T=\frac{1}{2} \sum_{i=1}^{6}\left(\rho_{i} A_{i} V_{i}^{2}+I_{x i} \omega_{x i}{ }^{2}+I_{y i} \omega_{y i}{ }^{2}+I_{z i} \omega_{z i}{ }^{2}\right)
$$

where $V_{i}{ }^{2}=\dot{X}_{i}{ }^{2}+\dot{Y}_{i}{ }^{2}+\dot{Z}_{i}{ }^{2} . I_{x i}, I_{y i}$ and $I_{z i}$ are the mass moments of inertia of the $i$-th element and are assumed to be equal for each wheel, each base, and each beam. Also, the effect of rotary inertia terms of the beams are ignored as in [25]. The combined $\rho A$ for the system can be calculated as:

$$
\rho A=\left\{\begin{array}{cc}
\rho_{b} A_{b}+\rho_{p} A_{p} & 0<x \leq L_{p} \\
\rho_{b} A_{b} & L_{p}<x \leq L
\end{array}\right.
$$

where $L$ and $L_{p}$ are the beam and the piezoelectric lengths, $\rho_{b}$ and $\rho_{p}$ are the densities of the beam and piezoelectric actuators, respectively, and $A_{b}$ and $A_{p}$ are the cross-sectional areas. Also, the potential energy of the beams and piezoelectric actuators can be expressed as:

$$
\begin{aligned}
& U=2 \rho g A L+\int_{0}^{L} \frac{1}{2} E_{b} I_{b}\left(\frac{\partial^{2} w_{1}}{\partial x_{1}{ }^{2}}\right)^{2} d x_{1} \\
& +\int_{0}^{L_{p}} \frac{1}{2} E_{p} I_{p}\left(\frac{\partial^{2} w_{1}}{\partial x_{1}{ }^{2}}+z_{p} d_{31} \frac{v_{1}(t)}{t_{p}}\right)^{2} d x_{1} \\
& +\int_{0}^{L} \frac{1}{2} E_{b} I_{b}\left(\frac{\partial^{2} w_{2}}{\partial x_{2}{ }^{2}}\right)^{2} d x_{2} \\
& +\int_{0}^{L_{p}} \frac{1}{2} E_{p} I_{p}\left(\frac{\partial^{2} w_{2}}{\partial x_{2}{ }^{2}}+z_{p} d_{31} \frac{v_{2}(t)}{t_{p}}\right)^{2} d x_{2} \\
& +\int_{0}^{L} \rho g A\left(-\sin \theta_{1} w_{1}+x_{1} \cos \theta_{1}\right) d x_{1} \\
& +\int_{0}^{L} \rho g A\left(-\sin \theta_{2} w_{2}+x_{2} \cos \theta_{2}\right) d x_{2}
\end{aligned}
$$

where $E_{b}$ and $E_{p}$ denote Young's modulus of elasticity of the beam and the piezoelectric, respectively, and $I_{b}$ and $I_{p}$ are the mass moments of inertia of the beam and piezoelectric crosssection about $y$-axis, respectively. In Eq. (16), $z_{p}$ is the neutral axis along the $z$-axis, $d_{31}$ denotes the piezoelectric constant of the actuator, $v_{1}(t)$ and $v_{2}(t)$ are voltages that are applied to the piezoelectric actuators and $t_{p}$ is the thickness of the piezoelectric actuators.

The damping effects of the beams can be taken to account as virtual work terms as follows:

$$
\delta W^{n c}=\int_{0}^{L} C_{1} \frac{\partial w_{1}}{\partial t} \delta w_{1} d x_{1}+\int_{0}^{L} C_{2} \frac{\partial w_{2}}{\partial t} \delta w_{2} d x_{2}
$$




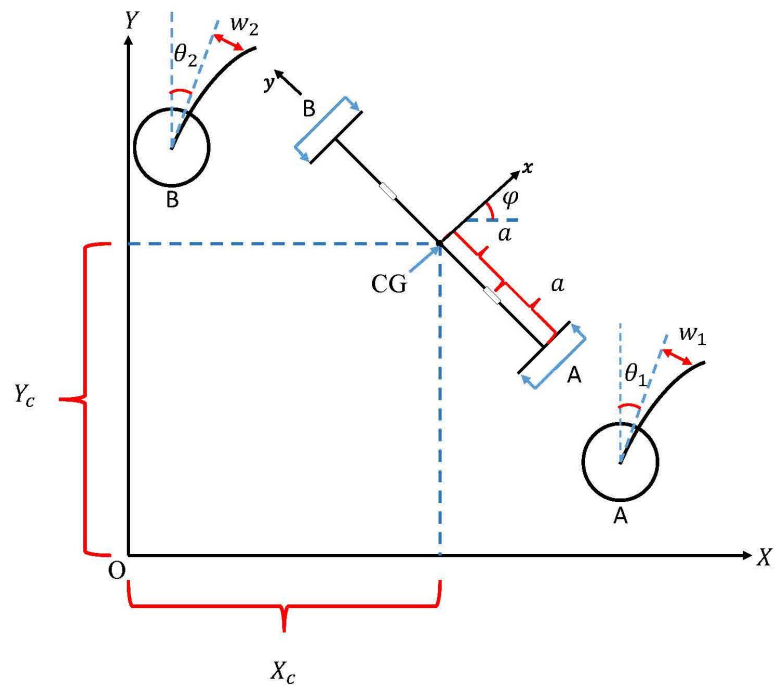

Fig. 2. The robot kinematics.

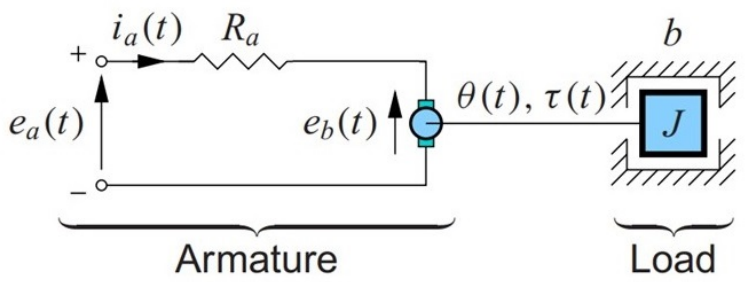

Fig. 3. The DC motor circuit diagram.

where $C 1$ and $C 2$ are the viscous damping coefficients of the beams. As noted, the robot is assumed to have two DC motors, which produce torques $\tau_{1}$ and $\tau_{2}$. The work of these external torques makes additional virtual work terms as:

$$
\begin{aligned}
& \delta W^{e x t}=\frac{\tau_{1}+\tau_{2}}{r_{w}}\left(\cos \varphi \delta X_{c}+\sin \varphi \delta Y+\left(Y_{c} \cos \varphi\right.\right. \\
& \left.\left.-X_{c} \sin \varphi\right) \delta \varphi\right)+F_{s}\left(-\sin \varphi \delta X_{c}+\cos \varphi \delta Y_{c}-\right. \\
& \left.\left(X_{c} \cos \varphi+Y_{c} \sin \varphi\right) \delta \varphi\right)
\end{aligned}
$$

In Eq. (18), the torques $\tau_{1}$ and $\tau_{2}$ are produced by the wheelconnected DC motors. The circuit diagram of the DC motors is shown in Fig 3. In this figure, the parameter $R_{a}$ denotes the armature resistance and the variables $V_{a}$ and $i_{a}$ are the applied voltage and the motor current draw, respectively. The equation of this system can be derived by applying the Kirchhoff's voltage Law to the circuit as:

$$
V_{a j}(t)=R_{a} i_{a j}(t)+\tau_{B j} \quad, j=1,2
$$

where $\tau_{B i}$ represents the back electromotive force (emf) and is equal to:

$$
\tau_{B j}=K_{B} \frac{\dot{X}_{w h e e l_{j}}}{r_{w}} \quad, j=1,2
$$

with $K_{B}$ being the motor speed coefficient. Here, torques $\tau_{1}$ and $\tau_{2}$ are given as:

$$
\tau_{j}=K_{t} i_{a j} \quad, j=1,2
$$

where $K_{t}$ is the motor torque constant and is provided by the manufacturer. Hence, the coupled equation can be obtained for the DC motor part by substituting Eq. (20) into Eq. (19).

$$
V_{a j}(t)=R_{a} i_{a j}(t)+K_{B} \frac{\dot{X}_{w h e e l_{j}}}{r_{w}} \quad, j=1,2
$$

The extended Hamilton's principle for this system can be written as:

$$
\int_{0}^{t}\left(\delta T-\delta U+\delta W^{n c}+\delta W^{e x t}\right) d t=0
$$

After substituting Eqs. (14)-(18) to Eq. (23) and some manipulations and simplifications, the dynamic equations of the system can be obtained as:

$$
\begin{aligned}
& \left(\frac{\tau_{1}+\tau_{2}}{r_{w}}\right) \cos \varphi-\sin \varphi F_{s}+\frac{2 I_{y_{w h e e l}}}{r_{w}^{2}}(\sin 2 \varphi \dot{X} \dot{\varphi}-\cos 2 \varphi \dot{Y} \dot{\varphi})-2\left(\frac{I_{y_{w h e e l}} \cos ^{2} \varphi+m_{w}{ }_{w}{ }^{2}}{r_{\text {base }}}\right) \ddot{X}+\frac{I_{y_{w h e e l}}}{r_{w}{ }^{2}} \sin 2 \varphi \ddot{Y}-\int_{0}^{L} \rho A\left[\ddot{X}+\left(a \cos \varphi-\cos \theta_{1} \sin \varphi w_{1}-x_{1} \sin \theta_{1} \sin \varphi\right)\right. \\
& \times \ddot{\varphi}_{1}+\left(-\cos \varphi \sin \theta_{1} w_{1}+x_{1} \cos \varphi \cos \theta_{1}\right) \ddot{\theta}+\cos \varphi \cos \theta_{1} \frac{\partial^{2} w_{1}}{\partial t^{2}}+\left(2 \sin \theta_{1} \sin \varphi w_{1}-2\right. \\
& \left.\times x_{1} \cos \theta_{1} \sin \varphi\right) \dot{\theta}_{1} \dot{\varphi}-2 \sin \varphi \cos \theta_{1} \dot{\varphi} \frac{\partial w_{1}}{\partial t}+\left(-a \sin \varphi-\cos \theta_{1} \cos \varphi w_{1}-x_{1} \cos \varphi \sin \theta_{1}\right) \\
& \left.\times \dot{\varphi}^{2}-2 \cos \varphi \sin \theta_{1} \dot{\theta}_{1} \frac{\partial w_{1}}{\partial t}+\left(-\cos \theta_{1} \cos \varphi w_{1}-x_{1} \cos \varphi \sin \theta_{1}\right) \dot{\theta}_{1}^{2}\right] d x_{1}-\int_{0}^{L} \rho A[\ddot{X}- \\
& \left(a \cos \varphi+\cos \theta_{2} \sin \varphi w_{2}+x_{2} \sin \theta_{2} \sin \varphi\right) \ddot{\varphi}-\left(\cos \varphi \sin \theta_{2} w_{2}-x_{2} \cos \varphi \cos \theta_{2}\right) \ddot{\theta}_{2}+\cos \varphi \\
& \times \cos \theta_{2} \frac{\partial^{2} w_{2}}{\partial t^{2}}+\left(2 \sin \theta_{2} \sin \varphi w_{2}-2 x_{2} \cos \theta_{2} \sin \varphi\right) \dot{\theta}_{2} \dot{\varphi}-2 \sin \varphi \cos \theta_{2} \dot{\varphi} \frac{\partial w_{2}}{\partial t}+(a \sin \varphi \\
& \left.-\cos \theta_{2} \cos \varphi w_{2}-x_{2} \cos \varphi \sin \theta_{2}\right) \dot{\varphi}^{2}-2 \cos \varphi \sin \theta_{2} \dot{\theta}_{2} \frac{\partial w_{2}}{\partial t}-\left(\cos \theta_{2} \cos \varphi w_{2}+x_{2} \cos \varphi\right. \\
& \left.\left.\times \sin \theta_{2}\right) \dot{\theta}_{2}^{2}\right] d x_{2}=0
\end{aligned}
$$


$\left(\frac{\tau_{1}+\tau_{2}}{r_{w}}\right) \sin \varphi+\cos \varphi F_{s}-\frac{2 I_{y_{w h e e l}}}{r_{w}{ }^{2}}(\sin 2 \varphi \dot{Y} \dot{\varphi}+\cos 2 \varphi \dot{X} \dot{\varphi})-2\left(\frac{I_{y_{w h e e l}}}{r_{w}{ }^{2}} \sin ^{2} \varphi+m_{w}\right.$ $\left.+m_{\text {base }}\right) \ddot{Y}-\frac{I_{y_{w h e e l}}}{r_{w}{ }^{2}} \ddot{X} \sin 2 \varphi-\int_{0}^{L} \rho A\left[\ddot{Y}+\left(a \sin \varphi+\cos \theta_{1} \cos \varphi w_{1}+x_{1} \cos \varphi \sin \theta_{1}\right)\right.$ $\times \ddot{\varphi}+\left(x_{1} \cos \theta_{1} \sin \varphi-\sin \theta_{1} \sin \varphi w_{1}\right) \ddot{\theta}_{1}+\cos \theta_{1} \sin \varphi \frac{\partial^{2} w_{1}}{\partial t^{2}}+\left(2 x_{1} \cos \theta_{1} \cos \varphi-2 \times\right.$ $\left.\cos \varphi \sin \theta_{1} w_{1}\right) \dot{\varphi} \dot{\theta}_{1}+2 \cos \theta_{1} \cos \varphi \dot{\varphi} \frac{\partial w_{1}}{\partial t}+\left(a \cos \varphi-\cos \theta_{1} \sin \varphi w_{1}-x_{1} \sin \theta_{1} \sin \varphi\right) \dot{\varphi}^{2}$ $\left.-2 \sin \theta_{1} \sin \varphi \dot{\theta}_{1} \frac{\partial w_{1}}{\partial t}+\left(-x_{1} \sin \theta_{1} \sin \varphi-\cos \theta_{1} \sin \varphi w_{1}\right) \dot{\theta}_{1}^{2}\right] d x_{1}-\int_{0}^{L} \rho A[\ddot{Y}-(a \sin \varphi$ $\left.-\cos \theta_{2} \cos \varphi w_{2}-x_{2} \cos \varphi \sin \theta_{2}\right) \ddot{\varphi}+\left(x_{2} \cos \theta_{2} \sin \varphi-\sin \theta_{2} \sin \varphi w_{2}\right) \ddot{\theta}_{2}+\cos \theta_{2} \sin \varphi$ $\frac{\partial^{2} w_{2}}{\partial t^{2}}+2 \cos \theta_{2} \cos \varphi \dot{\varphi} \frac{\partial w_{2}}{\partial t}+\left(2 x_{2} \cos \theta_{2} \cos \varphi-2 \cos \varphi \sin \theta_{2} w_{2}\right) \dot{\varphi} \dot{\theta}_{2}-2 \sin \theta_{2} \sin \varphi \dot{\theta}_{2}$ $\times \frac{\partial w_{2}}{\partial t}+\left(-a \cos \varphi-w_{2} \cos \theta_{2} \sin \varphi-x_{2} \sin \theta_{2} \sin \varphi\right) \dot{\varphi}^{2}+\left(-x_{2} \sin \theta_{2} \sin \varphi-\cos \theta_{2} \times\right.$ $\left.\left.\sin \varphi w_{2}\right) \dot{\theta}_{2}^{2}\right] d x_{2}=0$

$2 a\left(\frac{\tau_{1}-\tau_{2}}{r_{w}}\right)-\left(\frac{8 a^{2} I_{y_{w h e e l}}}{r_{w}^{2}}+8 a^{2} m_{w}+2 a^{2} m_{\text {base }}+2 I_{z_{w h e e l}}+2 I_{z_{\text {base }}}\right) \ddot{\varphi}+\frac{I_{y_{w h e e l}}}{r_{w}{ }^{2}} \sin 2 \varphi$ $\times \dot{Y}^{2}-\frac{I_{y_{w h e e l}}}{r_{w}{ }^{2}} \sin 2 \varphi \dot{X}^{2}+2 \frac{I_{y_{w h e e l}}}{r_{w}{ }^{2}} \dot{Y} \dot{X} \cos 2 \varphi-\int_{0}^{L} \rho A\left[\left(a \cos \varphi-\sin \varphi \cos \theta_{1} w_{1}-x_{1}\right.\right.$ $\left.\sin \varphi \sin \theta_{1}\right) \ddot{X}+a \cos \theta_{1} \frac{\partial^{2} w_{1}}{\partial t^{2}}+\left(a \sin \varphi+x_{1} \cos \varphi \sin \theta_{1}+w_{1} \cos \varphi \cos \theta_{1}\right) \ddot{Y}+\left(a^{2}+\right.$ $\left.x_{1}^{2}+\cos ^{2} \theta_{1} w_{1}^{2}-x_{1}^{2} \cos ^{2} \theta_{1}+x_{1} \sin 2 \theta_{1} w_{1}\right) \ddot{\varphi}+\left(a x_{1} \cos \theta_{1}-a \sin \theta_{1} w_{1}\right) \ddot{\theta}_{1}+\left(2 \cos ^{2} \theta_{1}\right.$ $\left.\times w_{1}+x_{1} \sin 2 \theta_{1}\right) \dot{\varphi} \frac{\partial w_{1}}{\partial t}-2 a \sin \theta_{1} \dot{\theta}_{1} \frac{\partial w_{1}}{\partial t}+\left(-a \cos \theta_{1} w_{1}-a x_{1} \sin \theta_{1}\right) \dot{\theta}_{1}^{2}+\left(x_{1}^{2} \sin 2 \theta_{1}\right.$ $\left.\left.-\sin 2 \theta_{1} w_{1}^{2}+2 x_{1} \cos 2 \theta_{1} w_{1}\right) \dot{\varphi} \dot{\theta}_{1}\right] d x_{1}-\int_{0}^{L} \rho A\left[-\left(a \cos \varphi+\sin \varphi \cos \theta_{2} w_{2}+x_{2} \sin \varphi\right.\right.$ $\left.\times \sin \theta_{2}\right) \ddot{X}+\left(a^{2}+x_{2}{ }^{2}+\cos ^{2} \theta_{2} w_{2}{ }^{2}-x_{2}{ }^{2} \cos ^{2} \theta_{2}+x_{2} \sin 2 \theta_{2} w_{2}\right) \ddot{\varphi}-\left(a \sin \varphi-x_{2} \cos \varphi\right.$ $\left.\times \sin \theta_{2}-\cos \varphi \cos \theta_{2} w_{2}\right) \ddot{Y}+\left(-a x_{2} \cos \theta_{2}+a \sin \theta_{2} w_{2}\right) \ddot{\theta}_{2}-a \cos \theta_{2} \frac{\partial^{2} w_{2}}{\partial t^{2}}+\left(a \cos \theta_{2}\right.$ $\left.\times w_{2}+a x_{2} \sin \theta_{2}\right) \dot{\theta}_{2}^{2}+\left(2 \cos ^{2} \theta_{2} w_{2}+x_{2} \sin 2 \theta_{2}\right) \dot{\varphi} \frac{\partial w_{2}}{\partial t}+2 a \sin \theta_{2} \dot{\theta}_{2} \frac{\partial w_{2}}{\partial t}+\left(x_{2}^{2} \sin 2 \theta_{2}\right.$ $\left.\left.-\sin 2 \theta_{2} w_{2}^{2}+2 x_{2} w_{2} \cos 2 \theta_{2}\right) \dot{\varphi} \dot{\theta}_{2}\right] d x_{2}=0$

$-I_{y_{b a s e}} \ddot{\theta}_{1}-\int_{0}^{L} \rho A\left[\left(w_{1}^{2}+x_{1}^{2}\right) \ddot{\theta}_{1}+\left(x_{1} \cos \theta_{1} \cos \varphi-\cos \varphi \sin \theta_{1} w_{1}\right) \ddot{X}+x_{1} \frac{\partial^{2} w_{1}}{\partial t^{2}}+\right.$ $\left(a x_{1} \cos \theta_{1}-a \sin \theta_{1} w_{1}\right) \ddot{\varphi}+\left(x_{1} \cos \theta_{1} \sin \varphi-\sin \theta_{1} \sin \varphi w_{1}\right) \ddot{Y}+2 w_{1} \dot{\theta}_{1} \frac{\partial w_{1}}{\partial t}\left(\sin \theta_{1} w_{1}^{2}\right.$ $\left.\left.\times \cos \theta_{1}-x_{1}^{2} \sin \theta_{1} \cos \theta_{1}-x_{1} \cos 2 \theta_{1} w_{1}\right) \dot{\varphi}^{2}-g w_{1} \cos \theta_{1}-x_{1} \sin \theta_{1}\right] d x_{1}=0$

$-I_{y_{\text {base }}} \ddot{\theta}_{2}-\int_{0}^{L} \rho A\left[\left(w_{2}^{2}+x_{2}^{2}\right) \ddot{\theta}_{2}+\left(x_{2} \cos \theta_{2} \cos \varphi-\cos \varphi \sin \theta_{2} w_{2}\right) \ddot{X}+x_{2} \frac{\partial^{2} w_{2}}{\partial t^{2}}-\right.$ $\left(a x_{2} \cos \theta_{2}-a \sin \theta_{2} w_{2}\right) \ddot{\varphi}+\left(x_{2} \cos \theta_{2} \sin \varphi-\sin \theta_{2} \sin \varphi w_{2}\right) \ddot{Y}+2 w_{2} \dot{\theta}_{2} \frac{\partial w_{2}}{\partial t}+\left(\sin \theta_{2}\right.$ $\left.\left.\times \cos \theta_{2} w_{2}^{2}-x_{2}^{2} \sin \theta_{2} \cos \theta_{2}-x_{2} \cos 2 \theta_{2} w_{2}\right) \dot{\varphi}^{2}-g w_{2} \cos \theta_{2}-g x_{2} \sin \theta_{2}\right] d x_{2}=0$ $\rho A\left(\frac{\partial^{2} w_{1}}{\partial t^{2}}+x_{1} \ddot{\theta}_{1}+a \cos \theta_{1} \ddot{\varphi}+\cos \theta_{1} \cos \varphi \ddot{X}+\cos \theta_{1} \sin \varphi \ddot{Y}-w_{1} \dot{\theta}_{1}^{2}+\left(-\cos ^{2} \theta_{1} w_{1}-\right.\right.$ $\left.\left.x_{1} \sin \theta_{1} \cos \theta_{1}\right) \dot{\varphi}^{2}\right)+C_{2} \frac{\partial w_{1}}{\partial t}+E I \frac{\partial^{4} w_{1}}{\partial x_{1}{ }^{4}}+\rho g A \sin \theta_{1}+\frac{\partial^{2}}{\partial x_{1}{ }^{2}}\left(\frac{E I S(x) z d_{31}}{t_{p}} V_{1}(t)\right)=0$ 


$$
\begin{aligned}
& \rho A\left(\frac{\partial^{2} w_{2}}{\partial t^{2}}+x_{2} \ddot{\theta}_{2}-a \cos \theta_{2} \ddot{\varphi}+\cos \theta_{2} \cos \varphi \ddot{X}+\cos \theta_{2} \sin \varphi \ddot{Y}-w_{2} \dot{\theta}_{2}^{2}+\left(-\cos ^{2} \theta_{2} w_{2}-\right.\right. \\
& \left.\left.x_{2} \sin \theta_{2} \cos \theta_{2}\right) \dot{\varphi}^{2}\right)+C_{1} \frac{\partial w_{2}}{\partial t}+E I \frac{\partial^{4} w_{2}}{\partial x_{2}}+\rho g A \sin \theta_{2}+\frac{\partial^{2}}{\partial x_{2}{ }^{2}}\left(\frac{E I S(x) z d_{31}}{t_{p}} V_{2}(t)\right)=0
\end{aligned}
$$

where

$$
S(x)=H(x)-H\left(x-L_{p}\right)
$$

and $H(x)$ is the Heaviside function. The boundary conditions of the above equations are represented as:

$$
\begin{aligned}
& \left(\frac{\partial^{2} w_{1}}{\partial x_{1}{ }^{2}} \delta \frac{\partial w_{1}}{\partial x_{1}}-\frac{\partial^{3} w_{1}}{\partial x_{1}{ }^{3}} \delta w_{1}\right)_{0}^{L}=0 \\
& \left(\frac{\partial^{2} w_{2}}{\partial x_{2}^{2}} \delta \frac{\partial w_{2}}{\partial x_{2}}-\frac{\partial^{3} w_{2}}{\partial x_{2}{ }^{3}} \delta w_{2}\right)_{0}^{L}=0
\end{aligned}
$$

To show the accuracy of Eqs. (24)-(33), it is sufficient to omit the $\varphi$ and one of these beams. Then, the final equations will convert to [23].

\section{NUMERICAL SIMULATION}

As described in the previous section, the derived equations of motion are complex and have many nonlinear and coupled terms. In this section, a solution technique for these nonlinear equations is briefly presented. The natural frequencies of the beams need to be obtained first in order to solve the obtained equations of motion and extract the natural frequencies of the whole system. In order to extract the natural frequencies of the beams, the following undamped, unforced equations of motion and boundary conditions of the beams are to be used:

$$
\begin{gathered}
\rho A \frac{\partial^{2} w}{\partial t^{2}}+E I \frac{\partial^{4} w}{\partial x^{4}}=0 \\
\left\{\begin{array}{c}
w(0, t)=0 \\
\frac{\partial w}{\partial x}(0, t)=0 \\
\frac{\partial^{2} w}{\partial x^{2}}(L, t)=0 \\
\frac{\partial^{3} w}{\partial x^{3}}(L, t)=0
\end{array}\right.
\end{gathered}
$$

Here, the beams are assumed to have harmonic motions with frequency $\omega$ as [26]:

$$
w=W(x) e^{i \omega t}
$$

Substituting Eq. (36) to (34), results the below equation:

$$
-\rho_{b} A_{b} \omega^{2} W+E I_{b} W^{\prime \prime \prime \prime}=0
$$

The general solution for 37 and its boundary conditions is given as

$$
W(x)=a_{1} \cos \beta x+a_{2} \sin \beta x+a_{3} \cosh \beta x+a_{4} \sinh \beta x
$$

where

$$
\beta^{2}=\sqrt{\frac{\rho A}{E I}} \omega
$$

Substituting boundary conditions (35) into eigenfunction (38), results in the following set of equations:

$$
A_{2 \times 2} X_{2 \times 1}=0
$$

where

$$
X_{2 \times 1}=\left[\begin{array}{l}
a_{1} \\
a_{2}
\end{array}\right]
$$

To obtain a nontrivial solution, the determinant of matrix $A$ in Eq. 40 should equal to zero. This equation gives the natural frequencies of the beams. The next step is to solve the equations of motion of the system using the assumed mode model expansion technique and the obtained natural frequencies. In this technique, the lateral displacements $w_{1}$ and $w_{2}$ are assumed as follows [27]:

$$
\begin{aligned}
& w_{1}=\sum_{i=1}^{\infty} W_{i}\left(x_{1}\right) q_{1 i}(t) \\
& w_{2}=\sum_{i=1}^{\infty} W_{i}\left(x_{2}\right) q_{2 i}(t)
\end{aligned}
$$

where $q_{1 i}(t)$ and $q_{2 i}(t)$ are the generalized coordinates for the bending of the beams and $W_{i}\left(x_{1}\right)$ and $W_{i}\left(x_{2}\right)$ are the mode shapes of a fixed-free beam. These functions are defined as:

$$
\begin{aligned}
& W_{i}\left(x_{i}\right)=A^{\prime}{ }_{1}\left(\sin \left(\beta_{n} x_{i}\right)-\sinh \left(\beta_{n} x_{i}\right)\right) \\
& +A^{\prime}{ }_{2}\left(\cos \left(\beta_{n} x_{i}\right)-\cosh \left(\beta_{n} x_{i}\right)\right)
\end{aligned}
$$

where $A_{1}^{\prime}$ and $A_{2}^{\prime}$ are two tunable and dependent coefficients as

$$
A^{\prime}{ }_{2}=-\frac{\left(\sin \left(\beta_{n} L\right)-\sinh \left(\beta_{n} L\right)\right)}{\left(\cos \left(\beta_{n} L\right)+\cosh \left(\beta_{n} L\right)\right)} A^{\prime}{ }_{1}
$$

with $\beta_{n}$ being defined as follows for each mode:

$$
\beta_{n}{ }^{4}=\frac{\rho_{b} \omega_{1 n^{2}}}{E I_{b}}
$$

The equations of motion can be obtained by substituting Eq. (42) to Eqs. (24)-(30) and also multiplying Eq. (43) to Eqs. (29) and 30, and then integrating the obtained equations over 0 to $L$. The final equations represent $2 n+5 \mathrm{DOF}$ of the system.

\section{Simulation Results}

To investigate the dynamic behavior of the system, the equations of motions are solved numerically in Matlab and the results are presented in different scenarios. The numerical values of the physical parameters are presented in Table I I and only two modes are considered for both beams. First, a sweep frequency input, shown in Fig 4, is applied to the system to extract the natural frequencies of the system. This standard input provides a fairly uniform spectral excitation and gives the modes of the systems [28]. Fig 5 shows the spectral analysis of time history of the system and its natural frequencies. 


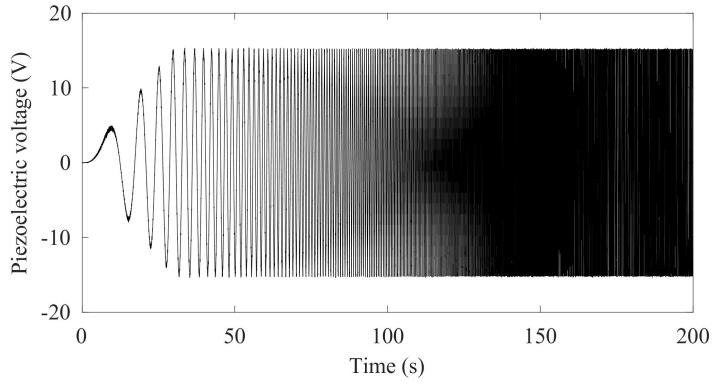

Fig. 4. The swipe frequency input voltage.

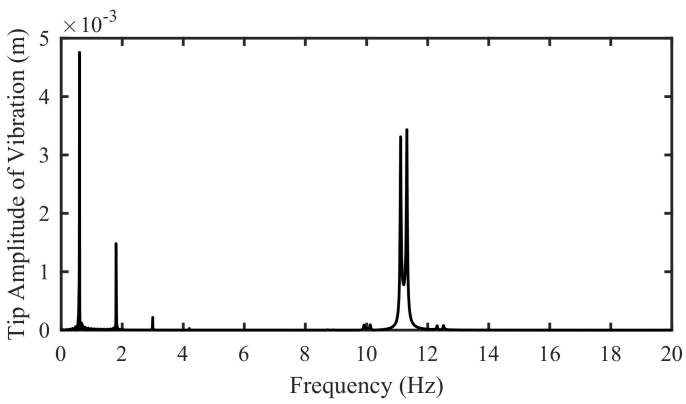

Fig. 5. The FFT analysis of the system.

In the next scenario, the lateral movement of the system in $X$-direction is presented by applying two same unit step input voltages to the piezoelectric actuators. It is assumed that the robot just moves forward. Hence, the below relation is set between the beams' rotation angle and the DC motors' voltage.

$$
\left\{\begin{array}{cc}
V_{a i}=10^{-2} \theta_{i} & \theta>0 \\
V_{a i}=0 & \theta<0
\end{array}, i=1,2\right.
$$

Fig 6 shows the lateral vibrations of the beams. These lateral vibrations, as shown in Fig 7 , make the beam rotation $\theta$ variations and consequently produce the DC motors voltages. As considered in the previous section, these voltages cause the external torques and the lateral movement in $X$-direction. The displacement of the robot is shown in Fig 8 Because of nonlinearity of the system, $\varphi$ and $Y$ are not zero but their size are small with respect to both $X$ and $\theta_{i}$ and considered negligible.

The final scenario is rotation test in the $X-Y$ plane. In this test, two step input voltages with different amplitudes are applied to the piezoelectric actuators. This makes different beams' lateral vibrations and bases' rotational angles, and consequently different torques applied to the wheels. This causes different angular velocities in the wheels and produces rotational angle $\varphi$ in the robot system. Fig 9 shows the rotation angles $\theta_{1}$ and $\theta_{2}$. The rotation and the path of the robot are also shown in Fig 10 and Fig 11 .

\section{CONCLUSIONS}

A new configuration for the conventional two-wheeled inverted pendulum system was presented in this paper. The
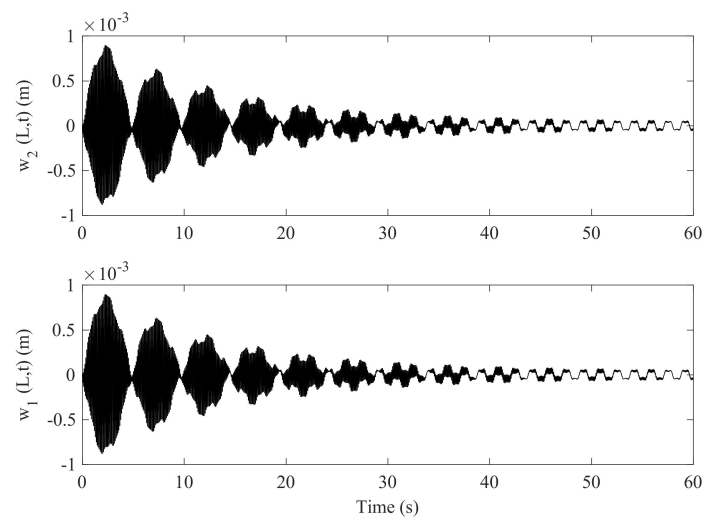

Fig. 6. Tip deflection of the beams $w_{1}(L, t)$ and $w_{2}(L, t)$ with unit step input.

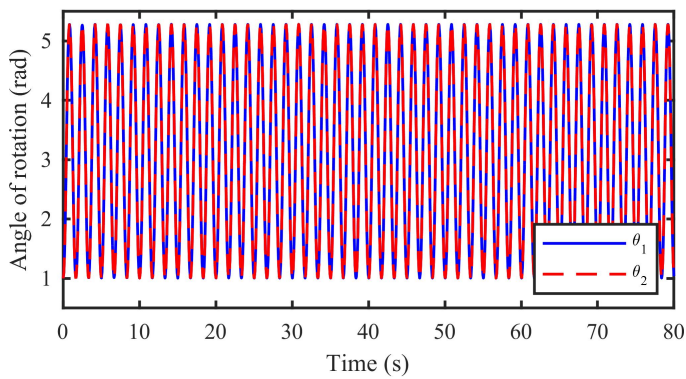

Fig. 7. Angular rotations of the beams $\theta_{1}$ and $\theta_{2}$ around the robot's base with unit step input.

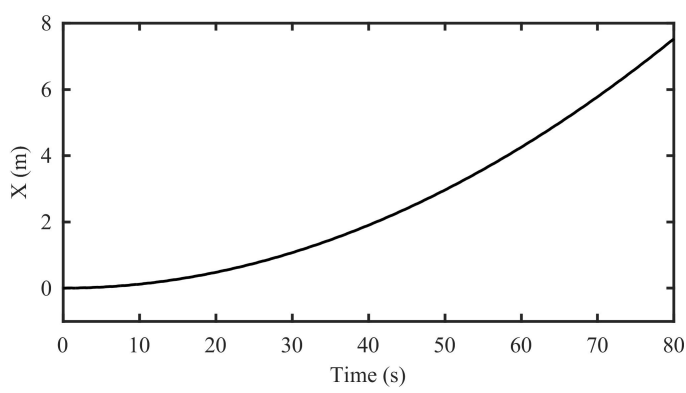

Fig. 8. Displacement of the robot in $X$-direction with step input.

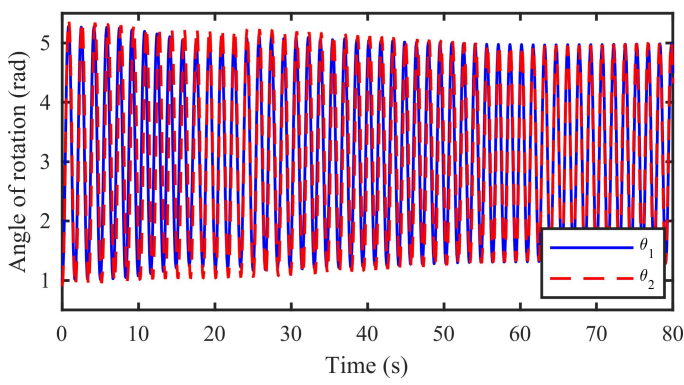

Fig. 9. Angular rotations of the beams $\theta_{1}$ and $\theta_{2}$ around the robot's base with $v_{1}=H(t)$ and $v_{1}=\frac{9}{10} H(t)$. 
TABLE I

THE SYSTEM PARAMETERS.

\begin{tabular}{llll}
\hline \hline Parameter & Value & Parameter & Value \\
\hline \hline Beam length $(\mathrm{mm})$ & 271.46 & Piezo layer shear modulus $(\mathrm{GPa})$ & 5.515 \\
Beam thickness $(\mathrm{mm})$ & 0.5 & Piezo layer elastic modulus $(\mathrm{GPa})$ & 30.33 \\
Beam width $(\mathrm{mm})$ & 25.65 & Piezo layer density $(\mathrm{Kg} / \mathrm{m} 3)$ & 5440 \\
Beam elastic modulus $(\mathrm{GPa})$ & 70 & First flexural damping ratio $(\%)$ & 0.0058 \\
Beam shear modulus $(\mathrm{GPa})$ & 30 & Second flexural damping ratio $(\%)$ & 0.015 \\
Piezo layer length $(\mathrm{mm})$ & 38 & PZT layer width $(\mathrm{mm})$ & 23 \\
Piezo layer thickness $(\mathrm{mm})$ & 0.3 & Beam density $(\mathrm{Kg} / \mathrm{m} 3)$ & 2700 \\
\hline
\end{tabular}

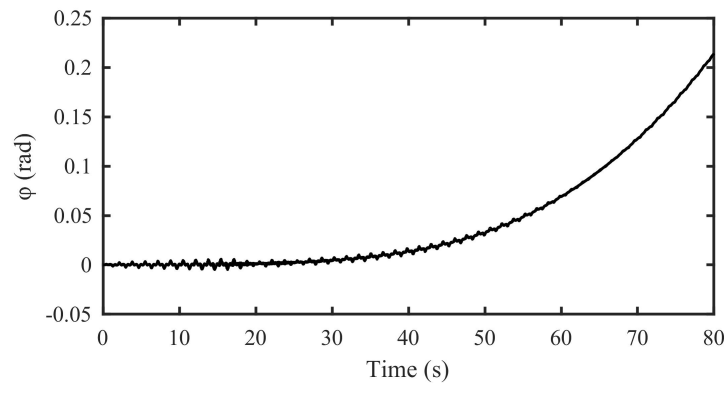

Fig. 10. Angular rotation $\varphi$ of the robot around the $z$-axis.

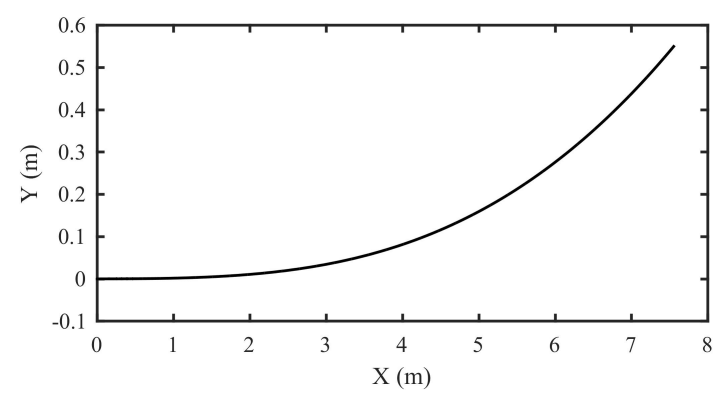

Fig. 11. Displacement $X$ and $Y$ of the robot in $X Y$-plane.

developed model had $2 n+5$ DOF and its main purpose was to simulate two cantilever beams and piezoelectric actuators on a moving base as a new robot which can move inplane. The governing equations of motion were obtained by employing the extended Hamilton's Principle. The derivation steps of these equations were presented in detail. The obtained model indicates that these systems have several coupled and nonlinear terms in their dynamics. To investigate the dynamic behavior of the system, these complex equations were solved numerically and the natural frequencies of the system were extracted. Finally, the result of two different tests including the lateral and circular movements were presented.

\section{REFERENCES}

[1] S. Jeong and T. Hayashi, "Development of a wheeled inverted pendulum mobile platform with a four-bar parallel mechanism," Advanced Robotics, vol. 32, no. 4, pp. 191-201, 2018.

[2] L. Canete and T. Takahashi, "Modeling, analysis and compensation of disturbances during task execution of a wheeled inverted pendulum type assistant robot using a unified controller," Advanced Robotics, vol. 29, no. 22 , pp. 1453-1462, 2015 .
[3] K. T. Ulrich, "Estimating the technology frontier for personal electric vehicles," Transportation research part C: Emerging technologies, vol. 13, no. 5-6, pp. 448-462, 2005.

[4] F. Grasser, A. D' arrigo, S. Colombi, and A. C. Rufer, "Joe: a mobile, inverted pendulum," IEEE Transactions on industrial electronics, vol. 49, no. 1, pp. 107-114, 2002.

[5] C.-C. Tsai, H.-C. Huang, and S.-C. Lin, "Adaptive neural network control of a self-balancing two-wheeled scooter," IEEE Transactions on Industrial Electronics, vol. 57, no. 4, pp. 1420-1428, 2010.

[6] J. Solis, R. Nakadate, Y. Yoshimura, Y. Hama, and A. Takanishi, "Development of the two-wheeled inverted pendulum type mobile robot wv-2r for educational purposes," in 2009 IEEE/RSJ International Conference on Intelligent Robots and Systems. IEEE, 2009, pp. 2347-2352.

[7] Double Robotics Inc, "Double 3," 2019. [Online]. Available: https: //Www.doublerobotics.com

[8] P. Frankovskỳ, L. Dominik, A. Gmiterko, I. Virgala, P. Kurylo, and O. Perminova, "Modeling of two-wheeled self-balancing robot driven by dc gearmotors," International Journal of Applied Mechanics and Engineering, vol. 22, no. 3, pp. 739-747, 2017.

[9] Y. Kim, S. H. Kim, and Y. K. Kwak, "Dynamic analysis of a nonholonomic two-wheeled inverted pendulum robot," Journal of Intelligent and Robotic Systems, vol. 44, no. 1, pp. 25-46, 2005.

[10] S. Kim and S. Kwon, "Dynamic modeling of a two-wheeled inverted pendulum balancing mobile robot," International Journal of Control, Automation and Systems, vol. 13, no. 4, pp. 926-933, 2015.

[11] M. Zafar and H. I. Christensen, "Whole body control of a wheeled inverted pendulum humanoid," in 2016 IEEE-RAS 16th International Conference on Humanoid Robots (Humanoids). IEEE, 2016, pp. 8994.

[12] M. Boukens and A. Boukabou, "Design of an intelligent optimal neural network-based tracking controller for nonholonomic mobile robot systems," Neurocomputing, vol. 226, pp. 46-57, 2017.

[13] S. Kim and S. Kwon, "Nonlinear optimal control design for underactuated two-wheeled inverted pendulum mobile platform," IEEE/ASME Transactions on Mechatronics, vol. 22, no. 6, pp. 2803-2808, 2017.

[14] M. Yue, C. An, and J.-Z. Sun, "An efficient model predictive control for trajectory tracking of wheeled inverted pendulum vehicles with various physical constraints," International Journal of Control, Automation and Systems, vol. 16, no. 1, pp. 265-274, 2018.

[15] A. Mehrvarz, A. Najafi Ardekani, M. J. Khodaei, and N. Jalili, "Vibration analysis and control of fluid containers using piezoelectricallyexcited side wall," Journal of Vibration and Control, vol. 25, no. 7, pp. 1393-1408, 2019.

[16] A. Mehrvarz, H. Salarieh, A. Alasty, and R. Vatankhah, "Vibration boundary control of timoshenko micro-cantilever using piezoelectric actuators," Scientia Iranica, vol. 25, no. 2, pp. 711-720, 2018.

[17] D. Karagiannis and V. Radisavljevic-Gajic, "Exponential stability for a class of boundary conditions on a euler-bernoulli beam subject to disturbances via boundary control," Journal of Sound and Vibration, vol. 446, pp. 387-411, 2019.

[18] B. Marzban and H. Yuan, "The effect of viscous force on the prediction of muscle contractility in biohybrid cantilever-based experiments," Extreme Mechanics Letters, vol. 9, pp. 342-346, 2016.

[19] A. Salehi-Khojin, N. Jalili, and S. N. Mahmoodi, "Vibration analysis of vector piezoresponse force microscopy with coupled flexurallongitudinal and lateral-torsional motions," Journal of Sound and Vibration, vol. 322, no. 4-5, pp. 1081-1099, 2009.

[20] M. Ansari, E. Esmailzadeh, and N. Jalili, "Coupled vibration and parameter sensitivity analysis of rocking-mass vibrating gyroscopes," Journal of Sound and Vibration, vol. 327, no. 3-5, pp. 564-583, 2009. 
[21] M. J. Khodaei, A. Mehrvarz, N. Candelino, and N. Jalili, "Theoretical and experimental analysis of coupled flexural-torsional vibrations of rotating beams," in ASME 2018 Dynamic Systems and Control Conference. American Society of Mechanical Engineers, 2018, pp. V003T42A004 V003T42A004.

[22] M. H. Nguyen, V. T. Ngo, M. T. Nguyen, T. T. H. Le, and V. Nguyen, "Designing linear feedback controller for elastic inverted pendulum with tip mass." Robotica \& Management, vol. 21, no. 2, 2016.

[23] A. Mehrvarz, M. J. Khodaei, W. Clark, and N. Jalili, "Modeling and dynamics analysis of a beam-hoverboard self-transportation system," in ASME 2018 Dynamic Systems and Control Conference. American Society of Mechanical Engineers, 2018, pp. V003T32A008-V003T32A008.

[24] W. R. Clark, "Control of a beam-hoverboard self-transportation system with model predictive control," Ph.D. dissertation, Northeastern University, 2019.

[25] V. Bhadbhade, N. Jalili, and S. N. Mahmoodi, "A novel piezoelectrically actuated flexural/torsional vibrating beam gyroscope," Journal of Sound and Vibration, vol. 311, no. 3-5, pp. 1305-1324, 2008.

[26] N. Jalili, Piezoelectric-based vibration control: from macro to micro/nano scale systems. Springer Science \& Business Media, 2009.

[27] S. S. Rao, Vibration of continuous systems. Wiley Online Library, 2007, vol. 464.

[28] A. Banazadeh, M. S. Seif, M. J. Khodaei, and M. Rezaie, "Identification of the equivalent linear dynamics and controller design for an unmanned underwater vehicle," Ocean Engineering, vol. 139, pp. 152-168, 2017. 